\title{
Conceptual, Spatial, and Cue Learning in the Morris Water Maze in Fast or Slow Kindling Rats: Attention Deficit Comorbidity
}

\author{
Hymie Anisman and Dan C. Mclntyre \\ Institute of Neuroscience, Carleton University, Ottawa, Ontario, Canada, K1S 5B6
}

Rat lines selectively bred for differences in amygdala excitability, manifested by "fast" or "slow" kindling epileptogenesis, display several comorbid features related to anxiety and learning. To assess the nature of the learning deficits in fast kindling rats, performance was evaluated in several variants of a Morris water-maze test. Regardless of whether the location of the platform was fixed or varied over days (matching-to-place task), the fast rats displayed inferior performance, suggesting both working and reference memory impairments. Furthermore, when the position of the platform was altered after the response was acquired, fast rats were more persistent in emitting the previously acquired response. The poor performance of fast rats was also evident in both cued and uncued tasks, indicating that their disturbed learning was not simply a reflection of a

Transgenic and knock-out mice have been used extensively in an effort to identify processes underlying pathological states (Crawley, 1999). However, because many pathologies involve multiple gene effects, or gene-environment interactions, an alternative approach has involved rat/mouse lines selectively bred to exhibit high or low levels of a given phenotype and then relating these to specific neuroanatomical, physiological, and chemical factors (Dudek and Underwood, 1993; Takahashi et al., 1994). To better understand temporal lobe seizures, we selectively bred two lines of rats for differences in amygdala excitability, as realized by either their "fast" or "slow" kindled seizure development (McIntyre et al., 1999a; Racine et al., 1999).

Not unexpectedly, comorbid features are often evident in selectively bred animals, likely reflecting either genetic pleiotropic or linkage effects (Parmigiani et al., 1999). In the fast and slow rats, comorbidities included heightened anxiety and stressor reactivity in slow rats, coupled with marked sexual impulsivity, impaired open-field habituation, and inferior delayed T-maze alternation acquisition in fast rats (possibly reflecting attention and associative deficits) (McLeod and McIntyre, 1995; Anisman et al., 1997, 2000; Mohapel and McIntyre, 1998; McIntyre et al., 1999b; Michaud et al., 1999; McIntyre and Anisman, 2000; Merali et al., 2001). The attention/associative deficits combined with impulsivity and hyperactivity are the defining features of human attention-deficit/hyperactivity disorder (ADHD) (American Psychiatric Association, 1994), a condition that often persists into adulthood (Faraone and

Received April 17, 2002; revised June 4, 2002; accepted June 17, 2002.

This work was supported by the Canadian Institutes of Health Research. H.A. holds a Canada Research Chair in Neuroscience. We are indebted to Charlene Dodd for her assistance.

Correspondence should be addressed to Hymie Anisman, Carleton University, Institute of Neuroscience, Life Science Research Building, Ottawa, Ontario K1S 5B6, Canada. E-mail: hanisman@ccs.carleton.ca.

Copyright (C) 2002 Society for Neuroscience $0270-6474 / 02 / 227809-09 \$ 15.00 / 0$ spatial deficit. Moreover, fast rats could be easily distracted by irrelevant cues, suggesting that these animals suffered from an attentional disturbance. Interestingly, when rats received several training trials with the platform elevated, permitting them to develop the concept of facile escape, the performance of fast rats improved greatly. The performance disturbance in fast rats may reflect difficulties in forming a conceptual framework under conditions involving some degree of ambiguity, as well as greater distractibility by irrelevant cues. These various attributes of the fast rats may serve as a potentially useful animal model of disorders characterized by an attention deficit.

Key words: seizure susceptibility; genetic differences; spatial learning; cued learning; concept formation; attention

Doyle, 2001). On the basis of these features, the focus of the present experiments was a more direct exploration of associative and attention problems in the fast compared with the slow rats.

The CNS differences between the fast and slow rats are widespread, being evident with respect to excitability differences in parahippocampal cortices (piriform and perirhinal) and the hippocampus (McIntyre et al., 1999a) and mesolimbic monoamine activity (McIntyre et al., 1999b; Anisman et al., 2000). As well, these rat lines differ with respect to seizure-induced neurotrophins, hippocampal granule cell sprouting, and $\mathrm{GABA}_{\mathrm{A}}$ subunit expression, all of which might contribute to their seizure predispositions (Kokaia et al., 1996; Elmér et al., 1998; Poulter et al., 1999). Because the integrity of the hippocampal system is important for spatial learning (Suzuki and Eichenbaum, 2000), the associative abilities in the rat lines were assessed in the Morris water-maze. Yet, because fast and slow rats were also thought to differ in attention (McLeod and McIntyre, 1995), there was no a priori reason to expect that performance disturbances in fast rats would be restricted to spatial tasks. Accordingly, performance was assessed in both spatial and cued paradigms. Finally, Morris water-maze performance involves several components, including concept formation (learning the general rules of the task), attention, working memory, and reference memory, which are not readily distinguishable in a simple form of this paradigm (Morris, 1989; Whishaw, 1989; Bannerman et al., 1995). Thus, we used several variants of the Morris water-maze in an effort to determine whether the rat lines differed in their (1) associative abilities, (2) conceptual abilities, (3) working and reference memory, (4) search strategies, and (5) native attention/distractibility.

\section{MATERIALS AND METHODS}

\section{Animals and housing conditions}

The procedures described in the present investigation were approved by the Carleton University Animal Care Committee and met all guidelines 
set out by the Canadian Council on Animal Care. Rats of the fast and slow lines were selectively bred from an original parent population composed of Wistar and Long-Evans Hooded rats. These lines, both of which are pigmented (hooded), were initially established at McMaster University (Hamilton, Ontario) through selective breeding without brother $\times$ sister matings. After the $F_{11}$ generation they were relocated to Carleton University, Institute of Neuroscience, where the differences in seizure susceptibility between these lines have remained despite relaxation of the selection procedure. Development of amygdala-kindled convulsions in fast rats normally requires $\sim 10$ trials, whereas 30 or more trials are required in slow rats (McIntyre et al., 1999a). Indeed, there is no overlap between the fast and slow rats with respect to the rate of development of amygdala kindling. The naïve rats of the present investigation were from the 43rd to 45 th generations of the two lines. The rats were tested between 3 and 4 months of age, were housed in pairs in standard opaque plastic cages $(32 \times 22 \times 20 \mathrm{~cm})$, maintained on a $12 \mathrm{hr}$ light/dark cycle (light phase: 7 A.M.-7 P.M.), and allowed ad libitum access to food and water. All testing was conducted between 8 A.M. and 12 P.M. to minimize behavioral variations attributable to diurnal rhythms.

\section{Apparatus}

Behavioral testing was conducted in a white, circular, polypropylene pool (158 cm in diameter, $60 \mathrm{~cm}$ height) that was filled with water $\left(21^{\circ} \mathrm{C}, 37.5\right.$ $\mathrm{cm}$ deep) made opaque by the addition of $\sim 2000 \mathrm{cc}$ of powdered milk. A clear Plexiglas, adjustable platform (35 $\mathrm{cm}$ height, $14 \mathrm{~cm}$ circumference), could be submerged $2.0 \mathrm{~cm}$ below the water surface or elevated $0.5 \mathrm{~cm}$ above the water level. In the case of cued training, unless specified otherwise, a proximal cue comprising a black cardboard in the shape of a cross $(10 \times 7 \times 2.5 \mathrm{~cm})$ hung above $(25 \mathrm{~cm})$ the platform. The pool was situated in a laboratory that contained assorted extra-maze cues, and the experimenter remained in the same position in the room throughout all testing trials. In some experiments, video recordings of the animals' performance was recorded by a camera situated on the ceiling, directly above the center of the pool. The videotape records were analyzed using a Smart Tracking System (San Diego Instruments, San Diego, CA).

\section{Behavioral procedures}

The simple Morris water-maze test requires that an animal, placed in different start positions, must find the location of a submerged platform. This simple response, however, may involve several components, some of which are not readily discerned in the typical Morris water-maze test. For instance, rats must initially learn the concept that a platform exists and that escape is possible. Additionally, once the platform is found they must maintain that memory over the short term, based on the platform's position during the immediately preceding trials (working memory), and over longer periods between days (reference memory) (Whishaw et al., 1995). Typically, the platform remains in the same location throughout the entire experiment, and hence working and reference memory are not readily distinguishable from one another. In a variant of this test, however, the position of the platform is altered between days but remains constant within each day (matching-to-place paradigm). Although rats are naïve about the platform's location on the first daily trial, the latency to find the platform nevertheless declines over days, as they learn the concept that a platform is located somewhere within the pool (i.e., reference memory). On any given day, once the platform is located, subsequent trials assess the rats' ability to recall the position of the platform (i.e., working memory) on that day (Whishaw, 1985). In the present investigation, both paradigms were used in an effort to identify the nature of the differences between the two rat lines.

In addition to differences in working and reference memory, various treatments may affect performance by influencing a wide range of associative and nonassociative factors. Among others, these include motoric abilities, concept formation, cue versus spatial learning, and behavioral flexibility or response perseveration, as well as attention or distractability. In the present investigation, several novel manipulations and paradigms were introduced to dissect the contribution of the various factors that might be responsible for the performance differences characteristic of the fast and slow rats.

Experiment 1: forced swim performance in the fast and slow rats. Because the two rat lines were to be assessed in a swim test that required relatively vigorous responding, a preliminary study was conducted to assess whether they would display differential active responding in a forced swim test. Rats $(n=5$ per line) received three trials of $3 \mathrm{~min}$ duration ( 1 min intervals between trials) in which they were placed in a clear, glass container with $21^{\circ} \mathrm{C}$ water. The container, which was $45 \mathrm{~cm}$ in height and had a diameter of $40 \mathrm{~cm}$, was filled with water to a height of $30 \mathrm{~cm}$. The behavior of the rats, which included swimming (four legs moving), upright struggling (two paws treading water and rats in a relatively vertical position) and floating, was recorded during each time period.

Experiment 2a-b: spatial learning in a fixed platform position paradigm. Rats $(n=8$ per line) received four trials in the Morris water-maze on each of $4 \mathrm{~d}$, without any cues signaling platform location. During testing, the submerged platform remained stationary in one quadrant of the maze, and the latency to find it (and the distance that rats swam) was determined. In this and all ensuing experiments, each trial consisted of an individual rat being placed carefully into the water, facing the outer edge of the pool, at one of four possible starting points (e.g., north, south, east, west). The starting location for each trial was determined randomly, with the provision that all start locations were used in a given day. A trial was terminated and the latency was recorded when the rat reached the platform and remained on it for $10 \mathrm{sec}$. If the rat did not reach the platform within $60 \mathrm{sec}$, the trial was terminated, and the rat was placed on the platform for $10 \mathrm{sec}$. Thereafter, rats were transferred to a dry holding cage where they remained for $60 \mathrm{sec}$ until the next trial. After training, rats were returned to their home cages. On the fifth day, rats received an additional $60 \mathrm{sec}$ probe trial in which a platform was not present within the pool. Rats were placed in the pool, as before, and the time spent within each of the quadrants of the pool was recorded over 15 sec periods, as were individual swim paths.

To further assess spatial learning in the fast and slow rats, a supplementary study (experiment $2 \mathrm{~b}$ ) was conducted in which initial place learning was followed by the platform position being altered to the diagonally opposite quadrant. Rats ( $n=6$ per line) were trained (four trials a day for $4 \mathrm{~d}$ ) as described in the preceding experiment. On the fifth day, the position of the platform was placed in the diagonally opposite quadrant, and performance was assessed for four trials after the procedures used during the preceding training days.

Experiment 3: spatial learning in a variable platform position paradigm (matching-to-place). It will be recalled that unlike the fixed-position paradigm, the matching-to-place test permits evaluation of both working and reference memory. In this study the procedure was the same as that described in the fixed-position paradigm, but with several exceptions. Because this task was somewhat more difficult to acquire than the fixed-position paradigm, rats $(n=10$ per line) received eight trials per day on each of 6 successive days. On each day, the platform was located in a new position (within the center of each of the four quadrants), with the provision that each location was represented no more than twice, and the position of the platform was not repeated within a $3 \mathrm{~d}$ period. On any given day, however, the position of the platform remained unchanged. Each trial, separated by 1 min intervals, commenced from one of the four starting locations, such that all four start positions were represented in the first four trials and again in the last four trials.

Experiment 4: cue learning in a fixed platform position paradigm. Although fast rats in the preceding experiments were found to exhibit impaired spatial learning, it was possible that they also encountered difficulties associated with stimulus-stimulus or stimulus-response relations. To assess the possibility that the fast and slow rats differ in a cued swim task, rats were assessed under conditions in which the location of the submerged platform was cued by an overhanging stimulus. Rats $(n=$ 8 per line) received $4 \mathrm{~d}$ of training (four trials per day) following the procedure used in the fixed-position paradigm described earlier. However, the platform position was signaled by the presence of an overhanging cue directly over the platform (a black cardboard cross $10 \times 7 \times 2.5$ $\mathrm{cm}, 25 \mathrm{~cm}$ above the water).

Experiment 5: cue learning in a variable platform position paradigm. In the preceding study, the platform position and cue were redundant (i.e., the cue and platform were always in a single location), and hence animals could have used either spatial or proximal cues to acquire the correct response. To differentiate whether the difference between fast and slow rats was genuinely related to proximal cue learning, an additional experiment assessed performance under conditions in which the platform position varied over trials of each day but was always paired with the overhanging cue. Rats $(n=11$ per line) received four trials on each of 4 consecutive days, where the position of the platform varied between the four quadrants on each trial of each day. Thus spatial cues could not be used to find the platform, and only the overhanging cue predicted its location. Other than the position of the platform and overhanging cue 
varying over days, the procedure was identical to that of the preceding study.

Experiment 6a-d: influence of pretraining on fixed and matching-to-place performance. In the noncued Morris water-maze, the rats had to find the submerged platform to escape from the water, with no previous training in the maze or assistance from proximal cues. Although the position of the platform was learned by both rat lines, the performance of the slow rats was markedly superior to that of the fast rats. As indicated earlier, a deficit in acquisition might reflect poorer working memory (i.e., remembering where the platform was on the previous trial that day), or it might reflect poorer reference memory (i.e., the rat might not recall the platform position from the previous day). Alternatively, it might reflect difficulties in acquiring the concept that a submerged platform exists. In view of the latter possibility, we again examined acquisition with the fixed- and variable-position procedure. However, in these instances, rats received pretraining with a raised platform, thereby permitting them to acquire the concept that an accessible platform existed.

In the first of these experiments, rats ( $n=6$ per line) were trained in a fixed-position paradigm for four trials as described earlier, except that the platform was raised and visible. On the ensuing $4 \mathrm{~d}$, the fixed-position procedure was continued, but the platform was submerged. In all other respects, the procedure was identical to the fixed-position paradigm described earlier.

In three additional experiments, rats $(n=8$ per line in each experiment) received $6 \mathrm{~d}$ of training in a noncued, variable-position paradigm, as described earlier. On the day preceding the initiation of this procedure, the two lines received either one, three, or eight trials of pretraining (in three experiments) with the raised platform.

Experiment 7: influence of nonspatial pretraining on performance in a matching-to-place task. Although pretraining rats with an elevated platform may serve to facilitate conceptual learning, thereby augmenting later performance, it is certainly possible that training rats with a visible platform in full view of distal stimuli may have facilitated performance by encouraging spatial learning (Morris, 1989; Whishaw et al., 1995). To assess this possibility, a procedure similar to that used by Morris (1989) was adopted in which rats were pretrained using an elevated platform; however, for one group, distal stimuli were present, whereas for a second group, distal stimuli were eliminated by a curtain that surrounded the pool. Fast and slow rats were assigned to one of the three pretraining conditions ( $n=9$ per group in each line). Rats in group 1 received no pretreatment, whereas those of group 2 received training for $1 \mathrm{~d}$ (eight trials) with a visible platform (as described in the preceding experiment). The third group also received training with the elevated platform, but the pool was surrounded by a floor-to-ceiling translucent curtain, thereby preventing rats from seeing distal cues. On the ensuing $4 \mathrm{~d}$, rats were tested in the matching-to-place task (eight trials per day). The testing procedure was essentially identical to that of the preceding experiments, except that the location of the platform on the four test days was either at the center of the pool, within the center of one the quadrants, or $20 \mathrm{~cm}$ from the pool wall. The platform location differed from that used in pretraining. Once again response latencies and swim path were recorded. A swim path was considered correct if rats swam directly to the platform (i.e., on a 30-cm-wide path).

Experiment 8: search strategies adopted under ambiguous conditions. The preceding studies suggest that fast and slow rats may have differed in their conceptual abilities. Thus, when the test situation was unambiguous (i.e., a platform was clearly visible), the differences between the rats was minimized. It was thus of interest to establish whether the rat lines differed in their search strategies in a relatively ambiguous situation. Thus, we assessed whether rats would display decreased response latencies (and search patterns) over a session under conditions in which the submerged platform was moved to a different part of the pool on each trial. In effect, the rats could not learn the position of the platform, and a decline of response latencies would likely reflect the development of some sort of search strategy or altered response style that culminated in improved performance.

Rats of the two lines ( $n=12$ per line) received a single day of training (eight trials) in which the position of the submerged platform varied between trials (center, along the perimeter $\sim 10 \mathrm{~cm}$ from the pool wall, or at the center of each quadrant). Trials were spaced 1 min apart, and the maximum duration of a trial was $60 \mathrm{sec}$. The start position varied over trials, so that each of the four start positions appeared twice. For half the rats, there were no proximal stimuli present, whereas an overhanging stimulus (as described previously) was present for the remaining rats but

\begin{tabular}{|c|c|c|c|}
\hline & Time 1 & Time 2 & Time 3 \\
\hline Fast & $167.60 \pm 1.03$ & $167.00 \pm 1.64^{*}$ & $162.40 \pm 3.88^{*}$ \\
\hline Slow & $157.20 \pm 7.33$ & $137.80 \pm 4.03$ & $123.60 \pm 10.06$ \\
\hline
\end{tabular}

${ }^{*} p<0.05$ relative to slow rats.

was unrelated to the platform position. Because the stimulus was without effect on performance, the data for the two conditions were pooled.

Experiment 9: influence of distractor stimuli on cued, variable-position performance. On the basis of earlier studies across various test situations (active and passive avoidance, habituation, delayed alternation), the possibility was raised that fast rats suffered from an impairment of attention (McIntyre and Anisman, 2000). If this was the case, then it might be expected that extraneous cues (distractor cues) would have a more profound disruptive effect on the fast compared with the slow rats. Rats of each line $(n=20$ per line) received 4 d of training (four trials per day) in the cued (overhanging stimulus) variable-platform position task described earlier; i.e., the platform position varied on each trial, and it was signaled by the overhanging cue $(6 \times 6 \times 15 \mathrm{~cm}$, black cardboard box). For half of the rats, no other cue was present, whereas for the remaining rats a second overhanging cue was present but was irrelevant to the platform position (distractor cue). A different distractor cue was used for each of the four daily trials that also appeared in a different location on each trial, to prevent the rat from learning that a particular stimulus signaled that the platform was not located beneath it. To this end, the four distractor cues were either a black and white striped $6.0 \mathrm{~cm}$ cube, a black and white plus sign, a striped 8-cm-diameter sphere, or an 8-cm-diameter black/white checkered ring.

\section{Statistical analyses}

The latency data for each of the experiments was subjected to mixed model ANOVAs with days and trials within days as within-group variables. All other variables were treated as between-group variables. The means comprising main effects and the means for simple effects of significant interactions, or for those interactions where a priori predictions had been made, were compared by Bonferonni corrected $t$ tests. When within-group comparisons were made, such as those involving days-to-trials effects, Tukey's comparisons were used. Analyses of the latencies to reach the platform during acquisition and the total distance rats swam were found to be highly correlated ( $r>0.90$ on most trials), and hence only latency data are presented. When the purpose of the experiment was to determine the specific swim path that the rats used, then the swim patterns are described.

\section{RESULTS}

\section{Experiment 1: forced swim performance in the fast and slow rats}

Active swimming varied as a function of the rat line $\times$ trials interaction $\left(F_{(2,16)}=3.90 ; p<0.05\right)$. The multiple comparisons indicated that during the first trial the two lines of rats did not differ from one another, with both spending almost all of their time engaged in active responses (Table 1). In the fast rats, active swimming remained unchanged throughout the three trials, whereas in slow rats it declined over the three trials and was replaced by floating.

\section{Experiment 2a-b: spatial learning in a fixed platform position paradigm}

Figure 1 shows the average latency (collapsed over the four trials each day) for the fast and slow rats to reach the fixed platform over each of $4 \mathrm{~d}$. The ANOVA showed that latencies declined over days in each of the lines $\left(F_{(3,42)}=18.37 ; p<0.01\right)$ and that the performance of slow rats was superior to that of fast rats $\left(F_{(1,14)}=24.59 ; p<0.01\right)$. By the last two trials of the fourth test day, both rat lines exhibited latencies of $<10 \mathrm{sec}$. Analyses of the videotape records did not reveal any specific search patterns 

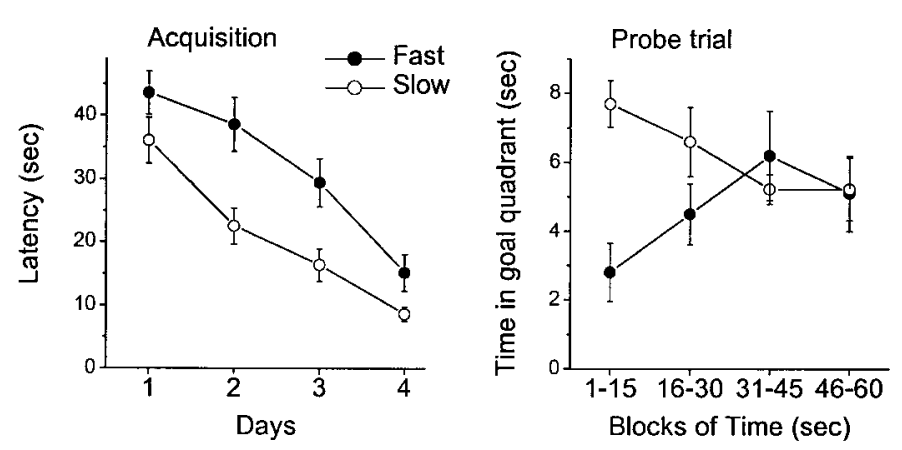

Figure 1. Mean $( \pm$ SEM) acquisition latencies (left panel) to reach the platform on four consecutive days (averaged over 4 trials per day) among fast and slow rats tested in a fixed position Morris water-maze paradigm. Time spent in the goal quadrant (i.e., the quadrant in which the platform had been located previously) over four $15 \mathrm{sec}$ periods is shown in the right panel.

unique to either rat line, which is not surprising given the rapid acquisition in both lines. The most notable difference between the lines was simply that during the initial test days slow rats were more likely to swim directly to the platform, whereas fast rats tended to make more directional or turning errors.

On the probe trial, the time that rats spent in the quadrant that had previously contained the platform varied as a function of the rat line $\times$ blocks of time interaction $\left(F_{(3,42)}=26.60 ; p<0.01\right)$. As shown in Figure 1 (right panel) and confirmed by the post hoc tests, the slow rats initially spent more time in this quadrant than did the fast rats, but this difference declined thereafter. In contrast, the remaining time spent in the quadrant increased in the fast rats. As a result, the initial between-group difference was absent during the second half of the probe trial. Examination of the probe trial video records indicated that of the eight slow rats, four swam directly to the platform (within a $30 \mathrm{~cm}$ band), whereas four approached the platform after making a relatively wide arc. In each instance, they remained in the goal quadrant for a few seconds before leaving this area. Of the fast rats, four swam directly to the platform, two made a wide arc in swimming to the platform, and two swam in the wrong direction. In contrast to the slow rats that lingered in the goal quadrant, all fast rats swam through the platform's previous location, and then when they reached the far wall of the pool they turned and left the quadrant. As a result, they initially spent little time in the quadrant. However, as reflected in Figure 1 (right panel), over the course of the trial, these fast rats tended to return to the location where the platform had been situated.

In the experiment assessing the effect of changing the platform position, initial acquisition was again found to improve over the $4 \mathrm{~d}\left(F_{(3,30)}=30.22 ; p<0.01\right)$, and the performance of Slow rats was superior to that of fast rats $\left(F_{(1,10)}=19.36 ; p<0.01\right)$. As shown in Figure 2 (left panel), however, by the fourth training day this difference was modest. On the fifth day, when the position of the platform was altered, performance was found to improve over trials $\left(\mathrm{F}_{(3,30)}=3.78 ; p<0.05\right)$, and the performance of slow rats was superior to fast rats. Figure 2, right-hand panel, shows the mean latencies on each trial on this test day. Interestingly, the performance of the fast rats (averaged over these four trials) was as poor as it had been on the first training day of initial acquisition. In contrast, the performance of slow rats was markedly superior to that evident on the initial training day. In effect, although the change in position resulted in negative transfer
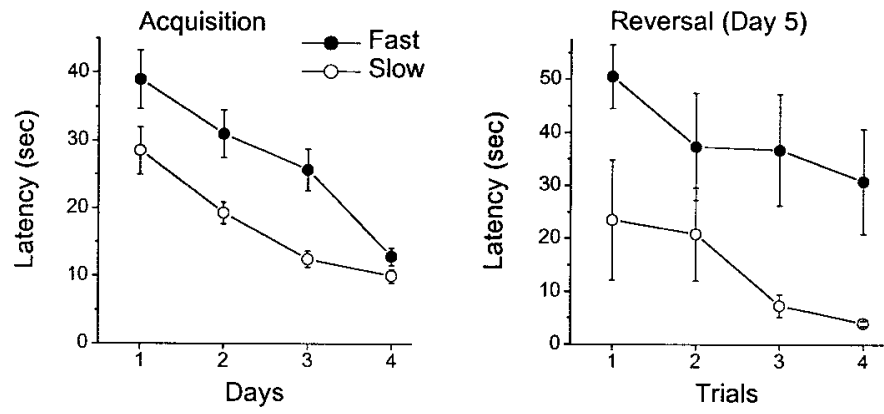

Figure 2. The left panel shows the mean $( \pm$ SEM) latencies to reach the platform on four consecutive acquisition days (averaged over 4 trials per day) among fast and slow rats tested in a fixed position Morris water-maze paradigm. The right panel shows the latencies on four consecutive trials among rats tested on a fifth day under conditions in which the platform was placed in a new location.

relative to that seen on the preceding day, the slow rats clearly gained from the experience of the preceding $4 \mathrm{~d}$. It is of interest, as well, that although all rats of both lines initially swam to the position of the platform, this tendency was more persistent in the fast rats. Indeed, throughout the four test trials (on day 5), all fast rats initially swam toward the position where the platform had been located previously. On the latter two trials, this tendency persisted, although once they swam past the previous platform location they soon found the new platform location. In the slow line, the tendency to swim toward the original platform location was seen only on the first two trials, after which all but one of these rats swam directly to the new location. Clearly, the fast rats, like their slow counterparts, had learned the response, but the fast rats were less likely to abandon this response even after the platform had been moved.

\section{Experiment 3: spatial learning in a matching-to-place paradigm}

Predictably, acquisition in the more difficult variable-platform position paradigm progressed more slowly than in the fixedposition paradigm. The ANOVA indicated that the latency to the platform varied as a function of the line $\times$ days $\times$ trials interaction $\left(\mathrm{F}_{(35,630)}=2.23 ; p<0.01\right)$. The multiple comparisons showed that performance improved over days and trials in both lines. However, performance among slow rats was superior to that of fast rats throughout the $6 \mathrm{~d}$ of testing (Fig. 3). On the initial trial of the first test day, the two rat lines exhibited similar response latencies. On that day, the performance of the slow rats improved markedly over the eight trials, with latencies falling to $\sim 15 \mathrm{sec}$ by the last three trials. In contrast, the fast rats exhibited inferior acquisition with very little improvement over trials. Latencies over trials declined more rapidly in the slow rats than in the fast rats. During the initial 2 test days, the superiority of the slow rats was clearly evident throughout the test session; this effect was slightly diminished during the next $4 \mathrm{~d}$, particularly during the later trials of each session as the fast rats eventually acquired the appropriate response.

It is particularly interesting that over days, the trial 1 performance of slow rats improved slightly, although the position of the platform varied from day to day, suggesting that these rats were acquiring the general concept that a platform existed or they were developing a more efficient search strategy. In contrast, there was little improvement on trial 1 performance over the $6 \mathrm{~d}$ in the fast rats (Table 2). 


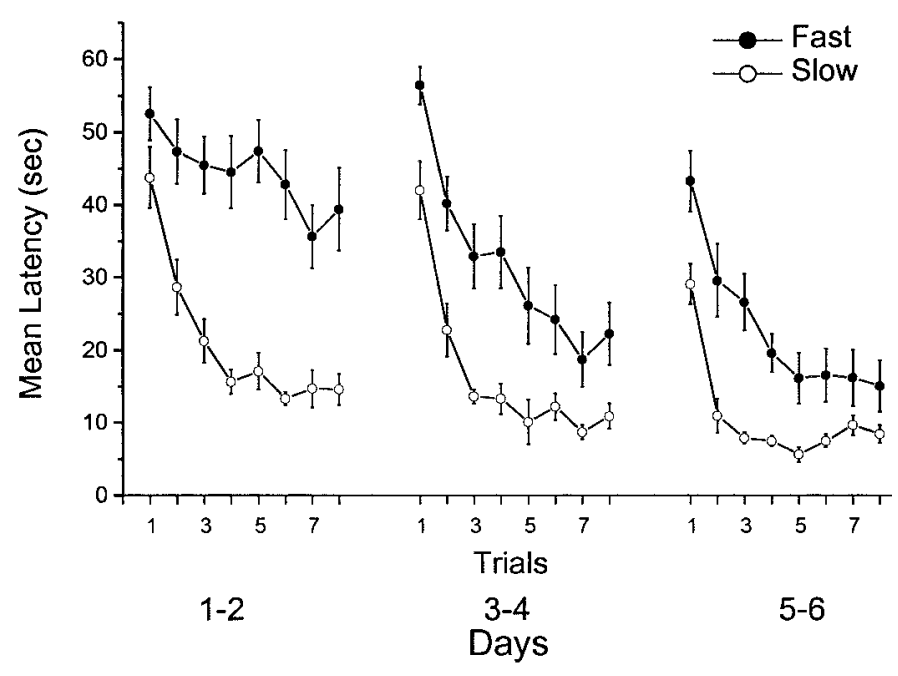

Figure 3. Mean ( \pm SEM) latencies to reach the platform on eight consecutive trials over $6 \mathrm{~d}$ (in $2 \mathrm{~d}$ blocks) in a matching-to-place paradigm among fast and slow rats. In this paradigm the position of the platform varied over days so that rats were required to remember the position of the platform based on the initial trial(s) of that day.

\section{Experiment 4: cue learning in a fixed platform position paradigm}

Performance in the cued task varied as a function of the line $\times$ days $\times$ trials interaction $\left(F_{(9,270)}=2.16 ; p<0.05\right)$. The multiple comparisons indicated that performance improved over days in both lines, but the performance of slow rats was superior to that of fast rats. On the first day, the superior performance of slow rats was only evident during the third and fourth trials, whereas on the ensuing $2 \mathrm{~d}$ the difference between the rat lines was apparent throughout all trials. On the last day of testing, in which all animals had essentially acquired the response, the rat line difference was limited to the first testing trial (Fig. 4, left panel).

\section{Experiment 5: cue learning in a variable platform position paradigm}

Figure 4 (right panel) shows the performance of the two rat lines in a cued paradigm in which the position of the platform changed on every trial. Changing the position of the platform on each trial of each day, while signaling the platform location, provided a method of assessing cued learning independent of spatial factors. In this test, the latencies to reach the platform were only moderately longer than they had been when the location of the platform was consistent across trials and days (compare Fig. 4). Latencies to reach the platform among slow rats again were shorter than that of fast rats $\left(F_{(1,20)}=17.78 ; p<0.01\right)$. Although performance improved over trials and days in both lines, the superiority of slow rats was maintained. However, as seen in Figure 4, by the fourth day the difference between the rat lines was small. Indeed, in this test, performance of the fast rats was far superior to that seen when the platform location was not cued (Fig. 3), attesting to the fact that the generally poorer performance of fast rats was not caused by visual impairments.

\section{Experiment 6a-d: influence of pretraining on performance in a fixed platform and a matching-to- place task}

If rats received four pretraining trials with an elevated platform (in a fixed platform position paradigm) and were tested subsequently with the platform submerged, both rat lines performed well, and the difference normally observed between them was entirely eliminated $(F<1)$. In this case, the latencies of fast rats on days $1-4$ were $35.67 \pm 3.97,11.63 \pm 1.46,7.87 \pm 0.99$, and $8.08 \pm 1.07$, whereas those of slow rats were $28.50 \pm 3.81,16.33 \pm$ $2.71,10.62 \pm 1.10$, and $10.16 \pm 1.08$. This result may be compared with the earlier experiments that showed substantial differences in latencies between the fast and slow rats in the fixed platform paradigm.

Pretraining with an elevated platform also appeared to minimize the differences between the rat lines tested in the matchingto-place test. Figure 5 shows the performance on days 1-6 (in blocks of $2 \mathrm{~d}$ ) among rats that received either one, three, or eight trials of pretraining with the raised platform. In rats that received only a single pretraining trial, the line differences were marked and sustained. The ANOVA indicated that performance varied as a function of the line $\times$ days $\times$ trials interaction $\left(F_{(35,490)}=1.57\right.$; $p<0.05)$. As observed in the earlier variable-platform position paradigm, where rats had not received pretraining, the multiple comparisons revealed that on the first trial of the first test day, the performance of the two lines was similar, but on the ensuing trials, performance improved more rapidly in the slow rats. As a result, the two lines differed markedly throughout the remainder of the test session. This difference was maintained throughout the 6 test days, although the difference was less marked on days 5 and 6, particularly during the later trials (Fig. 5, top panel).

When rats were given three pretraining trials with the elevated platform, subsequent acquisition latencies were still found to vary as a function of the line $\times$ trials interaction $\left(F_{(7,98)}=2.21 ; p<\right.$ $0.05)$. Generally, the line difference was least notable during the first trial of each day, became more pronounced on the intermediate trials, and was modest at the end of a session. The line $\times$ days interaction was not significant, but the performance of the two lines was similar on the last 2 test days. As seen in Figure 5 (middle panel), on the last 2 test days, the trial 1 latencies were moderately reduced relative to those observed on the first day, but on subsequent trials performance improved dramatically so that the difference between fast and slow rats was minimal. It is significant that comparisons between the rats that received one versus three pretraining trials with an elevated platform indicates that the latter rats gained appreciably more positive transfer than those rats that received only one such trial. Indeed, as seen in Figure 5, on days 3-4 of testing, the performance of rats that received three trials with the elevated platform was similar to the day 6 performance of rats that received only one trial.

Finally, after eight trials of pretraining with the elevated platform, performance in the submerged platform test varied as a function of the interactions between lines $\times$ days $\left(F_{(5,65)}=6.79\right.$; $p<0.01)$ and lines $\times$ trials $\left(\mathrm{F}_{(7,91)}=2.51 ; p<0.05\right)$. The multiple comparisons indicated that the performance of both lines improved over days and trials within each day. The difference between the lines was evident primarily on the initial 2 test days, but was absent thereafter, as the performance of the fast rats improved (Fig. 5, bottom panel). Importantly, even the trial 1 latencies declined dramatically over days, and by the last test day, the trial 1 latencies were very rapid, because animals had apparently learned the strategy that the submerged platform was located within a circumscribed area.

\section{Experiment 7: influence of nonspatial pretraining on performance in a matching-to-place task}

To assess whether distal cues contributed to the facilitative effects of pretraining with an elevated platform, rats received pretraining 
Table 2. Latencies of trial 1 on each of 6 consecutive days in a matching-to-place paradigm

\begin{tabular}{lllllll} 
& \multicolumn{1}{l}{ Day } & & & \\
\cline { 2 - 6 } & 1 & 2 & 3 & 4 & 5 & 6 \\
\hline Fast & $57.75 \pm 2.25$ & $52.38 \pm 6.41^{*}$ & $60.00 \pm 0.00^{*}$ & $52.38 \pm 6.21$ & $37.75 \pm 7.10$ \\
Slow & $52.25 \pm 6.64$ & $33.25 \pm 7.55$ & $29.25 \pm 7.71$ & $44.62 \pm 7.46$ & $39.62 \pm 7.71$ & $31.88 \pm 5.60$ \\
\hline
\end{tabular}

${ }^{*} p<0.01$ relative to slow rats.
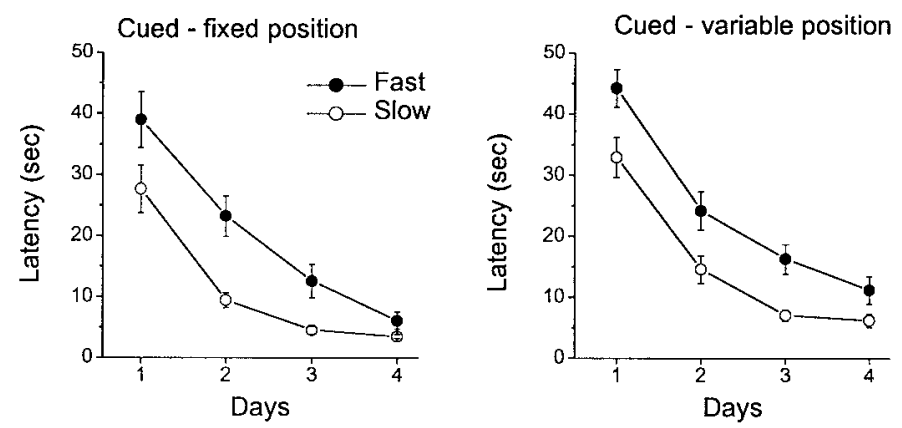

Figure 4. Mean ( \pm SEM) latencies to reach a platform cued by an overhanging stimulus on four consecutive days (averaged over 4 trials per day) among fast and slow rats. The cued platform was always in the same location (left panel) or varied on each trial of each day (right panel).

with distal cues visible or absent (the pool having been surrounded by a curtain during pretraining). On the pretraining day, during which latencies to reach an elevated platform were evaluated, there were no differences as a function of the rat line $(F<$ $1:$ mean $=19.75 \pm 1.64$ and $19.01 \pm 1.59$ for fast and slow rats, respectively) or as a function of whether distal cues were present or absent during training with the elevated platform $(F<1$ : mean $\pm \mathrm{SEM}=20.29 \pm 1.58$ and $18.47 \pm 1.65$ for rats tested with distal cues absent or present).

Figure 6 shows the latencies to reach the platform on each of eight trials (collapsed over the 4 test days) between fast and slow rats as a function of the pretraining condition. Response latencies in this paradigm varied as a function of the rat line $\times$ pretreatment condition $\times$ trials interaction $\left(F_{(14,336)}=2.26 ; p<0.01\right)$. The post hoc tests indicated that on the first daily trial, performance was comparable regardless of the rat line or the treatment condition. Among the slow rats the response was generally acquired quickly, and hence the performance-enhancing effects of the pretraining procedure were modest, being limited to trials $2-4$. In the absence of any pretreatment, the performance of the slow rats was superior to that of the fast rats. However, among pretrained fast rats, performance was superior to that seen among non-pretrained fast rats. Importantly, in both rat lines, pretraining enhanced performance, regardless of whether distal cues were present or absent. Thus, the experience with the elevated platform itself, rather than training in the context of distal cues, was primarily responsible for the superior performance observed.

Analysis of swim paths generally paralleled the latencies to reach the platform. Indeed, even on the first day of testing, the effects of the pretraining procedures were detectable in that correct responses (i.e., adopting a direct path to the platform) varied as a function of the rat line and the pretraining condition $\left(F_{(1,48)}=4.29, F_{(2,48)}=4.94 ; p<0.05\right)$. In the absence of pretraining, slow rats emitted more correct responses than did fast rats (mean $\pm \mathrm{SEM}=2.11 \pm 0.20$ and $1.00 \pm 0.37$, respectively). In slow rats, pretraining enhanced performance $(2.79 \pm$
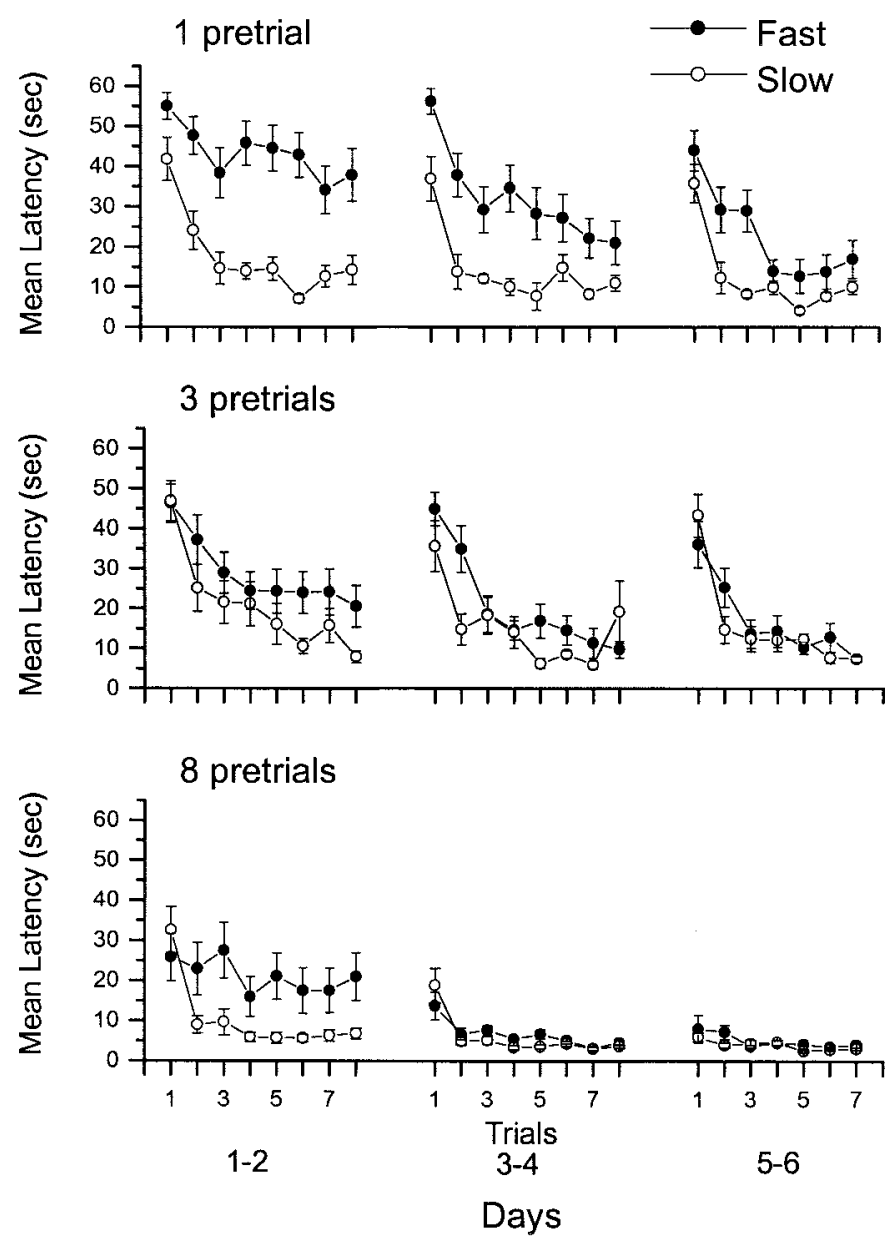

Figure 5. Mean $( \pm$ SEM) latencies to reach the platform on eight consecutive trials over $6 \mathrm{~d}$ (in $2 \mathrm{~d}$ blocks) in a matching-to-place paradigm among fast and slow rats. On the day before the initiation of the training rats received either one, three, or eight training trials in which the platform was elevated (top, middle, or bottom panel, respectively).

0.36 and $2.89 \pm 0.26$ among those trained with and without the curtain), and this effect was even more pronounced among fast rats $(2.22 \pm 0.57$ and $2.56 \pm 0.47$ with and without the curtain, respectively). Thus, it seems that pretraining with an elevated platform, regardless of whether distal cues were present, enhanced later performance in a matching-to-place test.

\section{Experiment 8: search strategies adopted under ambiguous conditions}

Fast and slow rats received eight training trials in which the position of the submerged platform varied over trials. Thus, the latency to find the platform stemmed from the rats either developing an effective search strategy (e.g., swimming in progressively smaller concentric circles or traversing the pool, as opposed to 

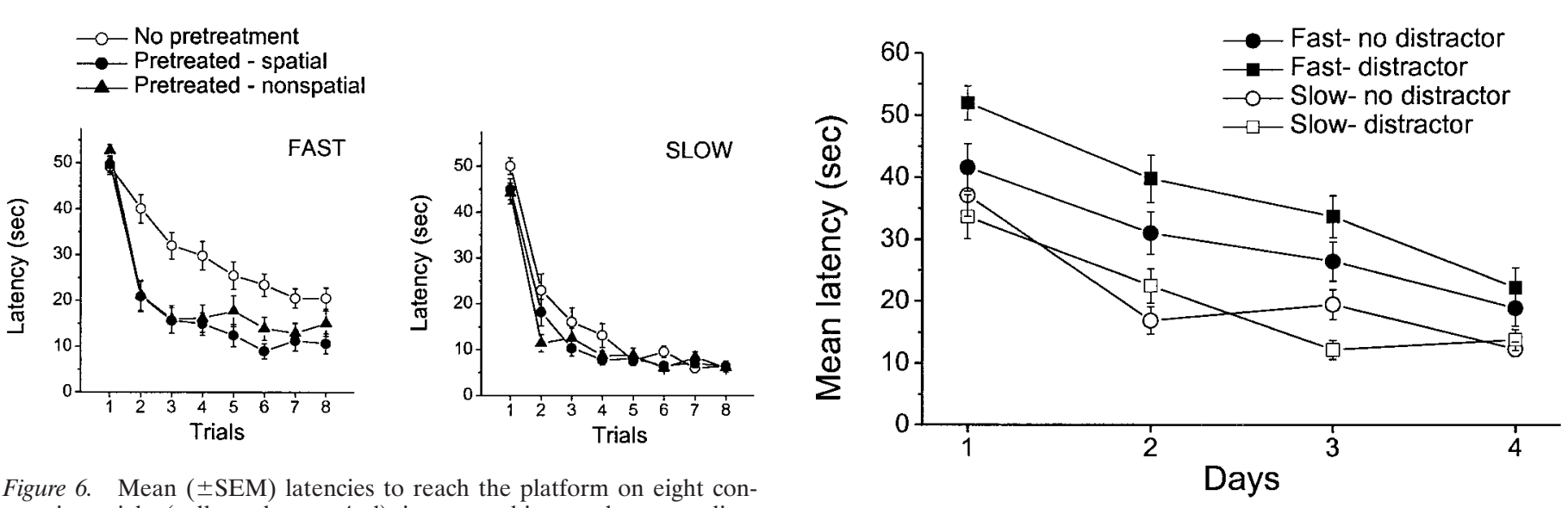

Figure 6. Mean $( \pm$ SEM) latencies to reach the platform on eight consecutive trials (collapsed over $4 \mathrm{~d}$ ) in a matching-to-place paradigm among fast (left) and slow (right) rats. On the day before the commencement of testing, rats of each line received either (1) no treatment, (2) eight training trials with an elevated platform and distal cues present, or (3) eight training trials with an elevated platform and distal cues absent (achieved by surrounding the pool with an opaque curtain).
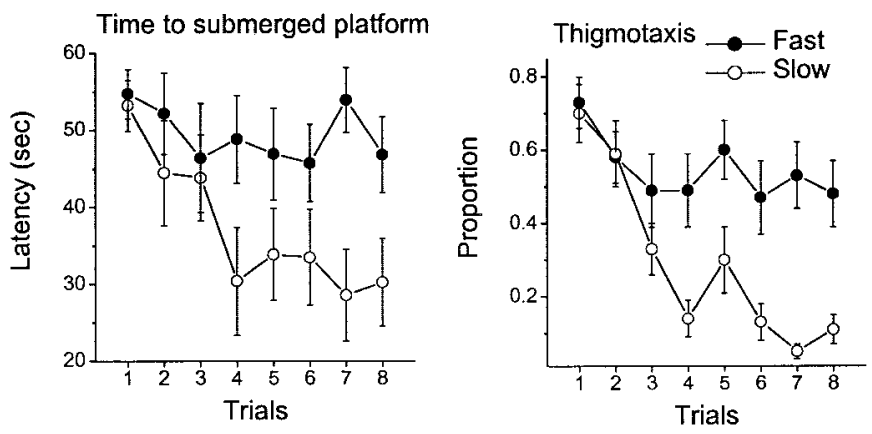

Figure 7. Mean $( \pm$ SEM) latencies to reach a submerged platform (left panel) and the thigmotaxic tendency (proportional distance swum along the pool perimeter relative to the total distance traversed; right panel) on eight consecutive trials. The location of the platform varied from trial to trial and was unsignaled; therefore, learning the specific platform location was not possible.

exhibiting thigmotaxic responses) or being more adept swimmers. The latencies of the slow rats to find the platform were significantly shorter than that of the fast rats $\left(F_{(1,20)}=15.24 ; p<0.01\right)$. The line $\times$ trials interaction was not significant, but the results over trials prove to be particularly informative. Specifically, as seen in Figure 7 (left panel), on the first three trials, performances of the two lines were essentially indistinguishable from one another. Thereafter, latencies in slow rats declined by $\sim 40 \%$ over the test session, whereas those of fast rats remained fairly stable. As a result, by the fourth trial, the difference between the lines reached statistical significance. Evidently, slow rats were gaining from the experience, or at least they were developing an effective search strategy, which did not appear to be the case for fast rats.

Inspection of the video records indicated that the two rat lines exhibited very clear differences in their search styles. Figure 7 (right panel) shows the distance that rats swam along the edge of the pool (i.e., within $10 \mathrm{~cm}$ ) as a proportion of the total swimming distance. The ANOVA indicated that swimming along the perimeter (measured as a proportion of distance swum along the perimeter over the total distance traversed) varied as a function of the rat line $\times$ trials interaction $\left(F_{(1,22)}=2.73 ; p<0.01\right)$. The multiple comparisons indicated that on the initial two trials, both rat lines favored a thigmotaxic response (swimming along the

Figure 8. Mean $( \pm$ SEM) latencies to reach the platform on 4 consecutive days (4 trials per day) in a cued variable-position paradigm among fast and slow rats. Rats of each line were tested under conditions in which only the cue signaling the platform position was present (no distractor) or in which the platform was signaled but another overhanging stimulus, acting as a distractor, was also present on each trial (distractor).

pool edge). Thereafter, however, the slow rats tended to make increasingly more pool traverses, and swimming along the pool edge declined. In contrast, although thigmotaxis also declined somewhat in the fast rats, they still continued to swim along the edge of the pool, and pool crossing occurred less frequently.

\section{Experiment 9: influence of a distractor stimulus on cued variable-position performance}

The latencies to reach the platform over the 4 test days for each of the two rat lines in the presence or absence of the distractor stimulus are presented in Figure 8. The ANOVA indicated that performance varied as a function of the line $\times$ distractor stimulus interaction $\left(F_{(1,36)}=6.62 ; p=0.01\right)$. Pairwise comparisons confirmed that among fast rats the presence of a distractor significantly increased the latencies relative to that seen in the absence of distractor cues. In contrast, the presence of distractor stimuli had no effect on the performance of slow rats. Although the effect of the distractor stimulus did not interact significantly with days, it appears from Figure 8 that the effect of the distractor was more notable during the initial days of testing. Clearly, in this experiment, the fast rats were more susceptible to the disruptive effects of distractor cues than were the slow rats.

\section{DISCUSSION}

It is not unusual for animals selected for a particular phenotype to exhibit comorbid characteristics remote to the selection criteria (Elias et al., 1975; Sandnabba, 1996; Gonzalez et al., 1998; Ferguson and Kennaway, 1999). In the present investigation, fast rats displayed marked acquisition impairments in a simple fixedposition spatial Morris water-maze, as well as in a test in which the position of the platform was altered after the response was acquired. Interestingly, on a probe trial, slow rats initially stayed in the area that previously contained the platform longer than fast rats, who swam through the platform area to the far wall and then engaged in an apparent random search. Despite the disposition of slow rats to initially remain in the vicinity of the previous platform, when it was moved, they readily abandoned the old location and rapidly acquired the new response. The fast rats, in contrast, persisted with previously learned responses and found the platform less readily. Parenthetically, these strain differences in performance were not likely caused by motoric factors, because fast 
rats were able to sustain active responses in a forced swim test as well as slow rats. Moreover, the performance difference was not attributable to greater stressor reactivity in fast rats, as slow rats typically display more profound anxiety-like responses, i.e., greater hypothalamic-pituitary-adrenal activation and amygdala corticotropin releasing hormone release in response to stressors (McIntyre et al., 1999b; Merali et al., 2001).

Ordinarily, spatial learning deficits associated with hippocampal disturbances are less evident when the position of the platform is cued by proximal stimuli (Aggleton et al., 1986). Because fast and slow rats differ with respect to hippocampal and parahippocampal excitability (McIntyre et al., 1999a) and hippocampal volume (Gilby and McIntyre, 2002), the possibility was considered that the behavioral deficits in fast rats might be of hippocampal network origin. However, when the position of the platform was proximally cued, regardless of whether it was fixed or variable across trials, performance of fast rats was still inferior to slow rats. Interestingly, the performance difference was absent when the platform was raised above the water. Apparently, as the overhanging cue was separated from the platform, rats had to make an association between the two cues, and some interpolation was necessary to acquire the concept that swimming to the overhanging cue led to escape. In contrast, when the platform was elevated and visible, the cue and safety were one and the same, minimizing the needed appraisal.

In addition to the poor performance in the simple spatial and cued tests, fast rats displayed markedly inferior performance in a matching-to-place task. In effect, having discovered the platform position on the first trial of each day, fast rats benefited less than slow rats, suggesting inferior working memory in the fast line. Interestingly, the lines not only differed with respect to betweentrials performance, but they were also distinguishable on the basis of their first trial response across days. In effect, unlike their slow counterparts, it appeared that fast rats were not acquiring or remembering the general concept of escape or applying it well over days. Furthermore, when the platform location was unsignaled and varied between trial on a given day, making it impossible to predict or learn its precise location, only the performance of slow rats improved over trials. The slow rats seemed to acquire the concept of escape and adopted strategies that improved performance (e.g., increased pool crossings). In contrast, fast rats seemed less able to acquire the concept and were more likely to maintain ineffective thigmotaxic responses rather than adopt more appropriate strategies. Thus, fast rats appeared behaviorally less flexible than slow rats.

It has been reported that although NMDA receptor-dependent long-term potentiation (NMDA-LTP) in the dentate gyrus is important for spatial learning (Moser et al., 1998), once the strategies necessary for learning are acquired, performance in spatial tasks progresses readily in the absence of NMDA-LTP (Bannerman et al., 1995; Saucier and Cain, 1995; Cain et al., 1996), depending on task difficulty and training procedures (Hoh et al., 1999). These data are commensurate with reports showing that limited pretraining diminishes the behavioral deficits ordinarily provoked by NMDA antagonists (Morris, 1989; Otnaess et al., 1999). Interestingly, the behavior in the water maze of naïve rats given NMDA antagonists (Cain et al., 1997; Whishaw and Auer, 1989; Hoh et al., 1999) was remarkably similar to our naïve fast rats. Specifically, not only did fast rats swim along the perimeter of the pool and fail to use effective search strategies, but when reaching the platform they often remained on it only momentarily. Importantly, when fast rats received pretraining with the raised platform, regardless of whether distal cues were present during pretraining and regardless of whether testing was conducted in a fixed-position or a matching-to-place paradigm, they appeared to acquire the conceptual strategies necessary for future learning, and deficits of reference and working memory were mostly eliminated. Indeed, as few as three pretraining trials were sufficient to augment the performance of fast rats, indicating that even limited familiarization with search strategies and conceptual training was sufficient for them to demonstrate their capability for spatial learning and memory. Clearly, if fast rats, like those treated with NMDA antagonists or fimbria-fornix lesions (Morris, 1989; Whishaw, 1989; Whishaw et al., 1995; Otnaess et al., 1999), did not learn the concept of escape or were deficient in developing good search strategies, they would appear, erroneously, to have severe working memory impairments. Alternatively, pretraining in fast rats might have improved performance by increasing their ability to attend to or focus on the critical features of the tasks.

In accordance with the suggestion that fast rats suffer from attention problems (McIntyre and Anisman, 2000), in a proximally cued test in which all rats were required to attend to the overhanging cue, the introduction of an irrelevant cue disrupted acquisition in fast rats but had no effect in slow rats. It seems that either fast rats were less able to differentiate the relevant from the irrelevant cues during acquisition or their attention was diverted more easily diverted from the relevant stimulus.

In summary, fast rats displayed marked behavioral impairments in a Morris water-maze test that appeared to encompass several distinct deficits. In this respect, the poor performance among fast rats was not limited to spatial tasks, being noticeable even when the position of the platform was signaled by an overhanging cue. Furthermore, fast rats seemed to suffer an attentional disturbance in that they were readily distracted by irrelevant stimuli. Moreover, unlike slow rats that seemed to adopt effective search strategies even when the platform location varied from trial to trial, fast rats abandoned thigmotaxic responses less readily and thus tended not to use effective search strategies. Yet the conceptual difficulties that fast rats appeared to endure were attenuated simply by giving them limited training with an elevated platform. Once rats acquired the concept that an accessible platform existed, performance disturbances were attenuated even in the fairly difficult matching-to-place test.

As in ADHD, in which impulsivity also is a common comorbidity (Hooper and Olley, 1996), another characteristic feature of fast rats is their disinhibited or impulsive behavioral style (McIntyre and Anisman, 2000). Such characteristics have been observed in various situations in which behavioral inhibition is absent in fast animals. This includes deficits of habituation in an open field and lack of response inhibition in an elevated plusmaze and a passive avoidance test (Mohapel and McIntyre, 1998). Furthermore, in a T-maze alternation test, fast rats ran down the alleyway as quickly as slow rats, indicating that they were well motivated to respond, but at the choice point they simply ran without hesitation in the direction in which they happen to be oriented (McLeod and McIntyre, 1995). Finally, in most rats, including the slow line, an estrous female will elicit sexual behaviors from the male, which are usually not forthcoming to a nonestrous female. The impulsive style of fast rats was apparent in their sexually assertive response to conspecific females, independent of the females' estrous state (Michaud et al., 1999). Invariably, fast males attempted to mount females shortly after their introduction and persevered in this behavior even when rebuffed. 
We have argued that fast rats may serve to model a syndrome such as ADHD. By themselves, the data of the present investigation do not speak to this syndrome. However, the behavior of these rats should be viewed in a broader context, including the fact that fast rats show diminished response inhibition and impulsivity. From such a perspective, the seizure-prone fast rats can provide a new animal model of ADHD with impulsivity. As already indicated, they show many attributes that define that eclectic human syndrome, including a predilection for seizures, which ordinarily is evident in up to $20 \%$ of ADHD individuals compared with $<2 \%$ of the normal population (Wolf and Forsythe, 1978). Ultimate validation of this model, however, will require pharmacological investigations that corroborate the human condition.

\section{REFERENCES}

Aggleton JP, Hunt PR, Rawlins JNP (1986) The effects of hippocampal lesions upon spatial and non-spatial tests of working memory. Behav Brain Res 19:133-146.

American Psychiatric Association (1994) Diagnostic and statistical manual of mental disorders, Ed 4. Washington, DC: American Psychiatric Association.

Anisman H, Lu ZW, Song C, Kent P, McIntyre D, Merali Z (1997) Influence of psychogenic and neurogenic stressors on endocrine and immune activity: differential effects in fast and slow seizing rat strains. Brain Behav Immun 11:63-74.

Anisman H, Kelly O, Hayley S, Borowski T, Merali Z, McIntyre DC (2000) Acoustic startle and fear potentiated startle in rats selectively bred for fast and slow kindling rates: relation to monoamine activity. Eur J Neurosci 12:4405-4416.

Bannerman DM, Good MA, Butcher SP, Ramsay M, Morris RG (1995) Distinct components of spatial learning revealed by prior training and NMDA receptor blockade. Nature 378:182-186.

Cain DP, Saucier D, Hall J, Hargreaves EL, Boon F (1996) Detailed behavioral analysis of water maze acquisition under APV or CNQX: contribution of sensorimotor disturbances to drug-induced acquisition deficits. Behav Neurosci 110:86-102.

Cain DP, Saucier D, Boon F (1997) Testing hypotheses of spatial learning: the role of NMDA receptors and NMDA-mediated long-term potentiation. Behav Brain Res 84:179-193.

Crawley JN (1999) Behavioral phenotyping of transgenic and knockout mice: experimental design and evaluation of general health, sensory functions, motor abilities, and specific behavioral tests. Brain Res 835:18-26.

Dudek BC, Underwood KA (1993) Selective breeding, congenic strains, and other classical genetic approaches to the analysis of alcohol-related polygenic pleiotropisms. Behav Genet 23:178-189.

Elias MF, Sorrentino RN, Pentz CA, Florini JR (1975) "Spontaneously" hypertensive mice: a potential genetic model for the study of the relationship between heart size and blood pressure. Exp Aging Res $1: 251-265$.

Elmér E, Kokaia M, Kokaia Z, McIntyre DC, Lindvall O (1998) Epileptogenesis induced by rapidly recurring seizures in genetically fast but not slow kindling rats. Brain Res 789:111-117.

Faraone SV, Doyle AE (2001) The nature and heritability of attentiondeficit/hyperactivity disorder. Child Adolesc Psychiatr Clin N Am 10:299-316.

Ferguson SA, Kennaway DJ (1999) Emergence of altered circadian timing in a cholinergically supersensitive rat line. Am J Physiol 277:R1171-R1178.

Gilby K, McIntyre DC (2002) Genetic mechanisms supporting fast and slow kindling. Spring Hippocampal Research Conference, Cayman Islands, April.

Gonzalez LE, File SE, Overstreet DH (1998) Selectively bred lines of rats differ in social interaction and hippocampal 5-HT1 receptor function: a link between anxiety and depression? Pharmacol Biochem Behav 59:787-792

Hoh T, Beiko J, Boon F, Weiss S, Cain DP (1999) Complex behavioral strategy and reversal learning in the water maze without NMDA receptor-dependent long-term potentiation. J Neurosci 19:RC2(1-5).

Hooper SR, Olley JG (1996) Psychological comorbidity in adults with learning disabilities. In: Adults with learning disabilities (Gregg N, Hoy C, Gay AF, eds), pp 162-183. New York: Guilford.

Kokaia Z, Kelly ME, Elmér E, Kokaia M, McIntyre DC, Lindvall O (1996) Seizure-induced differential expression of messenger RNAs for neurotrophins and their receptors in genetically fast and slow kindling rats. Neuroscience 75:197-207.

McIntyre DC, Anisman H (2000) Anxiety and impulse control in rats selectively bred for seizure sensitivity. In: Contemporary issues in modeling psychopathology (Mysblodsky M, Weiner I, eds), pp 29-45. New York: Kluwer Academic.

McIntyre DC, Kelly ME, Dufresne C (1999a) FAST and SLOW amygdala kindling rat strains: comparison of amygdala, hippocampal, piriform and perirhinal cortex kindling. Epilepsy Res 35:197-209.

McIntyre DC, Kent P, Hayley S, Merali Z, Anisman H (1999b) Influence of psychogenic and neurogenic stressors on neuroendocrine and central monoamine activity in fast and slow kindling rats. Brain Res 40:65-74

McLeod WS, McIntyre DC (1995) The effects of amygdala kindling on T-maze performance in epileptogenetically fast and slow kindling rat strains. Soc Neurosci Abstr 21:2115.

Merali Z, Kent P, Michaud D, McIntyre D, Anisman H (2001) Differential effects of psychogenic and neurogenic stressors on central corticotropin-releasing hormone and bombesin-like peptides in the fast and slow seizing rat. Brain Res 906:60-73.

Michaud D, McIntyre D, Anisman H, Merali Z (1999) Rat strains with high vs. low sexual reactivity: behavioral and lateralized amygdaloid CRH responses of males. Soc Neurosci Abstr 25:346.

Mohapel P, McIntyre DC (1998) Amygdala kindling-resistant (SLOW) or prone (FAST) rat strains show differential fear responses. Behav Neurosci 112:1402-1413.

Morris RG (1989) Synaptic plasticity and learning: selective impairment of learning rats and blockade of long-potentiation in vivo by the $N$-methyl-D-aspartate receptor antagonist AP5. J Neurosci 9:30403057.

Moser EI, Krobert KA, Moser MB, Morris RG (1998) Impaired spatial learning after saturation of long-term potentiation. Science 281:20382042.

Otnaess MK, Brun VH, Moser MB, Moser EI (1999) Pretraining prevents spatial learning impairment after saturation of hippocampal longterm potentiation. J Neurosci 19:RC49(1-5).

Parmigiani S, Palanza P, Rogers J, Ferrari PF (1999) Selection, evolution of behavior and animal models in behavioral neuroscience. Neurosci Biobehav Rev 23:957-969.

Poulter MO, Brown LA, Tynan S, Willick G, Williams R, McIntyre DC (1999) Differential expression of alpha 1, alpha 2, alpha, 3 and alpha 5 $\mathrm{GABA}_{\mathrm{A}}$ receptor subunits in seizure-prone and seizure-resistant rat models of temporal lobe epilepsy. J Neurosci 19:4654-4661.

Racine RJ, Steingart M, McIntyre DC (1999) Development of kindlingprone and kindling-resistant rats: selective breeding and electrophysiological studies. Epilepsy Res 35:183-195.

Sandnabba NK (1996) Selective breeding for isolation-induced intermale aggression in mice: associated responses and environmental influences. Behav Genet 26:477-488.

Saucier D, Cain DP (1995) Spatial learning without NMDA receptordependent long-term potentiation. Nature 378:186-189.

Suzuki WA, Eichenbaum H (2000) The neurophysiology of memory. Ann NY Acad Sci 911:175-191.

Takahashi JS, Pinto LH, Vitaterna MH (1994) Forward and reverse genetic approaches to behavior in the mouse. Science 264:1724-1733.

Whishaw IQ (1985) Formation of a place learning-set by the rat: a new paradigm for neurobehavioral studies. Physiol Behav 35:139-143.

Whishaw IQ (1989) Dissociating performance and learning deficits on spatial navigation tasks in rats subjected to muscarinic blockade. Brain Res Bull 23:347-358.

Whishaw IQ, Auer RN (1989) Immediate and long-lasting effects of MK-801 on motor activity, spatial navigation in a swimming pool and EEG in the rat. Psychopharmacology 98:500-507.

Whishaw IQ, Cassel JC, Jarrad LE (1995) Rats with fimbria-fornix lesions display a place response in a swimming pool: a dissociation between getting there and knowing where. J Neurosci 15:5779-5788.

Wolf SM, Forsythe A (1978) Behavioral disturbance, phenobarbital, and febrile seizures. Pediatrics 61:728-731. 